\title{
Systematic Approach to the Formation of Cluster Structures and Enterprises in Agro-Industrial Complex of the Voronezh Region
}

\author{
Bukhovets A.G.* \\ Voronezh State Agricultural University named after \\ Emperor Peter the Great \\ Voronezh, Russia \\ E-mail: abuhovets@mail.ru
}

\author{
Gorelova M.V. \\ Voronezh State Agricultural University named after \\ Emperor Peter the Great \\ Voronezh, Russia
}

\author{
Shishkina L.A. \\ Voronezh State Agricultural University named after Emperor Peter the Great \\ Voronezh, Russia
}

\begin{abstract}
The work is devoted to the analysis of cluster structures and enterprises in the agro-industrial sector of the Voronezh region from the point of system analysis view. In the framework of the systematic approach, the formed cluster structures have rank distributions corresponding to the hyperbolic Zipf's law. It is shown that the hyperbolic laws with a real indicator characterize formations with a systemic organization. The consideration of the formed cluster structures as system objects leads to the need to assess the shape of rank distributions. To verify the fulfillment of the systematic conditions of the analyzed agro-industrial cluster, the method of statistical tests was used. The article presents the results of testing on the data of the agro-industrial cluster in the Voronezh region, which allow coming to the conclusion about the nature of the ranking distribution. It is shown that the newly formed cluster of agricultural enterprises demonstrates the necessary properties of system formation. The fulfillment of the Zipf distribution on the constructed rank distribution confirms the assumption of the systemic nature of the new economic structure. In general, the results obtained indicate the perspectives of a new economic structure.
\end{abstract}

Keywords - system analysis, hyperbolic rank distributions, cluster structures, agribusiness of the Voronezh region.

\section{INTRODUCTION}

The importance of a system approach to the organization of new functional systems is primarily determined by the fact that such formations are integral objects that in practice demonstrate resistance to external and internal disturbances, as well as the ability to adapt effectively to changing conditions, showing the ability to self-organize.

As a necessary condition for systemacity, it is proposed to consider the fulfillment on a set of objects - elements of the system which the Zipf rank distribution [4, 9] can be represented in the following form

$$
n_{i}=\frac{C}{i^{\gamma}}
$$

where $i=1,2, \ldots, K$ is the rank of the class; $\mathrm{C}$ is a constant value, usually equal to the volume of the largest class; $n_{i}$ is the volume of class $i$ rank; $\gamma$-a certain constant positive value having a value greater than unity $(\gamma=1+\alpha, 0<\alpha \leq 1)$.

The formation mechanisms of this distribution, also known as the Pareto distribution, have been considered in a number of works (see, for example, [3, 4] and others). As a part of our approach, we will consider the system as some fractal set [1], for which the property of self-similarity is specific. We show that under these conditions the rank distribution (1) is fulfilled with necessity.

\section{METHODS AND MATERIALS}

Suppose that there is a certain set of objects in some linear space $\mathrm{R}^{\mathrm{p}}$, and $k$ is a certain size characterizing this set. Let $k^{\prime}$ be another scale value at which a self-similar structure is observed. Let $S(k)$ be some property that depends on the magnitude of the selected scale. The presence of the selfsimilarity property of structures for the indicated scales allows writing

$$
\frac{S(k)}{S\left(k^{\prime}\right)}=f\left(\frac{k}{k^{\prime}}\right)
$$

that is, the ratio of the properties $S(k)$ and $S\left(k^{\prime}\right)$ is a function of the selected scales ratio. Considering successively the values of $k, k^{\prime}$ and $k^{\prime \prime}$ and calculate the following relations:

$$
\frac{S(k)}{S\left(k^{\prime}\right)}=f\left(\frac{k}{k^{\prime}}\right), \quad \frac{S\left(k^{\prime}\right)}{S\left(k^{\prime \prime}\right)}=f\left(\frac{k^{\prime}}{k^{\prime \prime}}\right), \quad \frac{S(k)}{S\left(k^{\prime \prime}\right)}=f\left(\frac{k}{k^{\prime \prime}}\right),
$$

which allow obtaining equality

$$
\frac{S(k)}{S\left(k^{\prime}\right)} \frac{S\left(k^{\prime}\right)}{S\left(k^{\prime \prime}\right)}=f\left(\frac{k}{k^{\prime}}\right) f\left(\frac{k^{\prime}}{k^{\prime \prime}}\right) .
$$

Introducing the following notation

$$
\frac{S(k)}{S\left(k^{\prime \prime}\right)}=f\left(\frac{k}{k^{\prime \prime}}\right)=f\left(\frac{k}{k^{\prime}}\right) f\left(\frac{k^{\prime}}{k^{\prime \prime}}\right),
$$

we obtain 


$$
f\left(\frac{k}{k^{\prime \prime}}\right)=f\left(\frac{k}{k^{\prime}} \frac{k^{\prime}}{k^{\prime \prime}}\right)=f\left(\frac{k}{k^{\prime}}\right) f\left(\frac{k^{\prime}}{k^{\prime \prime}}\right) .
$$

The functional equation, which in more familiar notation can be represented as

$$
f(x y)=f(x) f(y)
$$

This equation, in which the function $f$ acts as the unknown, belongs to the 4 type of Cauchy functional equations. The General solution of this equation can be written as

$$
f(x)=x^{\gamma},
$$

where $\gamma$ is an arbitrary real number. Since (3) it is supposed to be used to characterize rank distributions in which the classes of objects are ordered by decreasing their number, it is convenient to assume that $\gamma=1+\alpha, \alpha>0$, and represent the function in the form

$$
f(x)=\frac{C}{x^{1+\alpha}}
$$

It is easy to see that in this case Zipf's law will be obtained in the form (1).

Thus, the manifestation of power function laws with a real exponent is a consequence of some kind of self-similarity (scaling) - a characteristic feature of sets with a systematic organization.

\section{METHODOLOGY}

The methodology for verifying the fulfillment of the Zipf's law on the rank distribution of the objects structure, proposed by us in $[3,4]$, is based on a statistical test of the hypothesis about the value of the parameter $\gamma=1+\alpha$ in the relation (1). For this, we take the logarithm of the expression (1) and obtain the linear equation with respect to the estimated parameter $\gamma$ :

$$
\ln \left(n_{i}\right)=\ln (C)+\gamma \ln (i) .
$$

Then, in a standard way, the parameters of the obtained regression equation are estimated using the least squares method, for example, using the STATISTICA package [7].

Verification of the fulfillment of the Zipf distribution is reduced to statistical verification of the main hypothesis $H_{0}: \gamma<1$, with the competing $H_{1}: \gamma \geq 1$. In this case, the null hypothesis corresponds to a uniform distribution with a given number of classes. Comparison with a uniform distribution is due to the fact that it is this distribution that gives the maximum of entropy, a function that characterizes the ordering degree of the set under consideration. In practical problems, it is convenient to use the duality principle of estimating the parameters values of the regression model using confidence intervals [10].

To verify the assumptions formulated above, the simulation method was used. It consisted in constructing a rank partition of a set with a predetermined number of classes, the numbers of which had a uniform distribution. Using the rank distribution obtained, the parameter $\gamma$ was estimated. Then this procedure was repeated a sufficiently large number of times (in our case, for example, 1000). Based on the statistical estimation of the distribution density of the parameter $\gamma$ values, the critical field for the null hypothesis was determined from the given significance level. Such an approach made it possible to construct an interval in which, with a given reliability, the value of the ranking distribution parameter of interest is located.

Further testing of the hypothesis $H_{0}$ is carried out in a standard way. To implement the approach represented above, we developed a program in the MathCad system, where the Rnd program was used as a pseudo-random number sensor.

\section{RESULTS AND DISCUSSION}

As it is shown above, the exercise of hyperbolic laws with a real exponent is a consequence of some kind of properties common to the system organization of the analyzed objects sets. The establishment of the systematic nature of the data under study provides certain grounds for believing that we are dealing with processes that are based on self-organization mechanisms.

Our proposed methodology for verifying the fulfillment of the Zipf's law was used to analyze the cluster structure of enterprises forming the agro-industrial cluster of the Voronezh region [5]. In total, according to official data [8], the agroindustrial cluster includes 36 enterprises of various forms of ownership of LLC, ZAO and AOO. For analysis, the following indicators of economic activity such as current assets, non-current assets, stocks, revenue, cost, profit (loss), net profit, number were taken [2].

A statistical values analysis of these indicators shows that a significant scatter characterizes these data. This generally agrees well with the assumption of the hyperbolic nature of the Zipf rank distribution.

To test the statistical hypothesis about the type of rank distribution, i.e. the relation fulfillment (1), a preliminary analysis of the rank distributions of all analyzed indicators was previously carried out. Hyperbolic dependence is traced in all signs. As an example, the most clearly defined dependence for the "Current assets" indicator is presented in Figure 1.

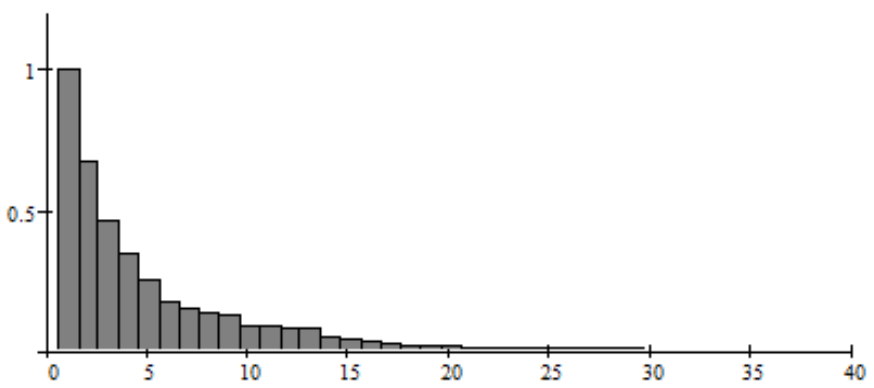

Fig. 1. The ranking distribution of the «Current assets" indicator

A more detailed statistical analysis was carried out using the methodology previously discussed. To do this, the data were preliminarily taken and then a regression equation was constructed in twice-logarithmic form coordinates.

As an example, we present the results of the "Current assets" indicator analysis (V2), which characterizes the 
systemic nature of the cluster. Note that, as the results of the correlation matrix analysis show, all the signs taken are significantly correlated at the standard $5 \%$ level, which allows, in our opinion, to use with equal success any of the presented signs.

The regression equation constructed in twice-logarithmic coordinates will have the form

$$
\ln (\mathrm{V})=16,0347-2,3152 * \ln (\mathrm{N}), \quad\left(\mathrm{R}^{2}=0,87\right)
$$

where $\mathrm{V}$ is a selected indicator for ranking; $\mathrm{N}-$ the object rank in the list of objects ordered by attribute $\mathrm{V}$; $\ln \left(^{*}\right)$ is a logarithmic function, the basis of which has no fundamental value.

The constructed equation by $87 \%$ explains the regression dependence between the variables, which indicates a good statistical quality of the equation.

Taking into account the obtained simulation results, it is possible to construct a region of acceptance of the null hypothesis at a given level of $95 \%$ reliability. For our case, it will be represented by an interval $(-1,102 ;-0,547)$. The $95 \%$ confidence interval for the rank distribution parameter at the same significance level, calculated based on the results of the regression equation calculation, will be $(-2.573 ;-2.067)$, which is in good agreement with the assumption of the value of the indicator $\gamma>1$.

The results indicate that the available data do not contradict the hypothesis of the cluster structure existence at a $5 \%$ significance level. In other words, the assumption expressed above about the nature of the systemic organization of the agribusiness enterprises complex in the Voronezh region is confirmed in the data presented with a reliability exceeding $95 \%$.

\section{V.CONCLUSION}

The implementation of the Zipf distribution on the rank distribution of the agro-industrial cluster in the Voronezh region may indicate the prospects of a new economic structure. Newly formed enterprise cluster in the agroindustrial complex demonstrates the necessary properties of systemic formation. It can be assumed that this structure previously developed independently, because of selforganization processes, and subsequently received the formal status of an agrarian cluster.

\section{References}

[1] A.G. Bukhovets, Modeling data structures in classification problems. Germany: Palmarium Acad. Publ., 2012, 247 p. ISBN: 978-3-65998107-4.

[2] A.G. Bukhovets et al., "On the identification of systemic properties of cluster structures in the agro-industrial complex of the Voronezh region", Bull. of the Voronezh State Agrar. Univer., vol. 4, iss. 41-42, pp.203-210, 2016

[3] A.G. Bukhovets, "System interpretation of classification results", Sociol.: methodol., methods, mathemat. Models, vol. 22. pp. 114-144, 2006.

[4] A.G. Bukhovets, "A systematic approach and rank distributions in classification problems / A.G. Bukhovets", Bull. of the Voronezh State Univer., ser. Econ. and Manag. vol. 1, pp. 130-142, 2005.

[5] On approval of the concept of cluster policy in the Voronezh region in the industrial sector of the economy, Order of the Department of Industry, Transport and Innovation of the Voronezh Region, dated 04.25.2012, vol. 67

[6] Russian cluster observatory. Retrieved from: http://cluster.hse.ru

[7] A.A. Khalafyan, statistica 6, Statistical data analysis, Textbook. Moscow: Binom-Press LLC, 2010, 528 p.

[8] Cluster Development Center of the Voronezh Region. Retrieved from: http://www.cluster36.ru

[9] Yu.A. Schreider, Systems and models, Textbook. Moscow: Radio and communicat, 1982, $152 \mathrm{p}$.

[10] L.P. Yanovsky, Introduction to Econometrics, Textbook. Moscow: Knorus, 2017, 256 p. 